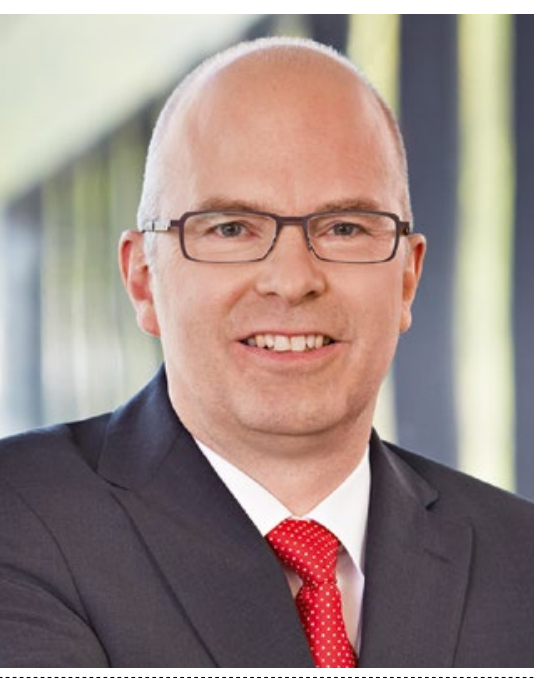

Dr.-Ing. Rainer Otterbach ist Leiter Produktmanagement bei der dSpace GmbH in Paderborn.

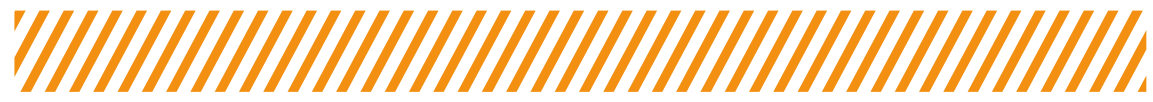

\title{
„Ein Knackpunkt liegt im Datenmanagement“
}

ATZelektronik_Herr Dr. Otterbach, was bedeutet es für Sie, mit der enorm steigenden Komplexität und den weiter steigenden Kosten in der Softwareentwicklung umzugehen?

OTTERBACH _ Wir bieten Kunden mit unseren Werkzeugen die Möglichkeit, noch mehr zu simulieren und zu virtualisieren. So können sie früher mit der Validierung ihrer Systeme beginnen und noch mehr Tests von der Straße ins Labor verlegen.

Dann tun Sie das, was Sie immer schon getan haben, nur intensiver. Wo liegen die Potenziale in der Absicherung?

Wenn unsere Kunden von virtueller Absicherung reden, meinen sie heute meistens die HiL-Simulation, um Tests von der Straße ins Labor zu holen. Mit der reinen Softwaresimulation gehen wir noch einen Schritt weiter. Dabei werden Steuergeräte als virtuelle Prototypen verwendet, die auf einem PC in einer simulierten Umgebung getestet werden. Darin liegt das große Potenzial, weil Abhängigkeiten und das Zusammenspiel vieler Komponenten sehr früh getestet werden können, lange bevor ein Hardwareprototyp vorliegt.

Wird die Absicherung mit virtuellen Steuergeräten bereits bei Autoherstellern eingesetzt?

Ja. Es laufen Pilotanwendungen in ersten Serienprojekten. Ein deutscher Automobilhersteller ist bereits sehr weit und konsequent in der Anwendung. Dort hat man auf Basis virtueller Steuergeräte einen zusätzlichen Absicherungsschritt eingeführt, um die Applikationssoftware zusammen mit der Basissoftware früh im Closed-Loop-Betrieb zu validieren.

Firmen wie dSpace haben mit HiL- und SiLSystemen eine revolutionäre Entwicklungsumgebung geschaffen. Ist eine zweite Revolution in den kommenden zehn Jahren zu erwarten? Das vernetzte und hochautomatisierte Fahren sowie die Elektromobilität stellen hohe Anforderungen an die Testsysteme. Deshalb wollen wir das Testen mit virtuellen Steuergeräten als zusätzlichen Absicherungsschritt neben der Echtzeitsimulation am HiL-Simulator etablieren. Dabei zeichnet sich auch ein Trend zu heterogenen Entwicklungsumgebungen ab, wo Prüfstände und Simulatoren je nach Testaufgabe flexibel zusammengeschaltet werden. Das lässt sich nur mit aufeinander abgestimmten Testlösungen bewältigen.

Wie werden Sie die bevorstehende Datenflut in den Griff bekommen?

Mit einem Datenmanagement, was unter anderem die Wiederverwendung von Simulationsmodellen und Tests erleichtert. Das erfordert neben den Veränderungen in den Entwicklungsprozessen und gegebenenfalls Anpassungen in der Organisation auch das entsprechend passende Datenmanagementsystem.

\section{Gibt es hier einen Knackpunkt?}

Ja. Die heutigen Werkzeuge des Application Lifecycle Managements und des Product Lifecycle Managements können nicht alle Artefakte der modellbasierten Entwicklung und ihre Abhängigkeiten untereinander in der notwendigen Detailtiefe abbilden. Man benötigt zusätzliche Werkzeuge, mit denen man zum Beispiel bei der Entwicklung eines sicherheitskritischen Steuergeräts die Beziehungen zwischen Anforderungen und den daraus abgeleiteten Funktionsmodellen und Tests darstellen kann. Dazu muss das Datenmanagementsystem aber wissen, was ein Modell und was ein Signal ist, oder was Testfälle und Testdaten sind.

\section{Demnach ist mehr gefragt als nur eine} dateibasierte Ablage. Gibt es so etwas schon? Wir bieten ein derartiges Datenmanagementsystem an, das bereits produktiv bei Kunden im Einsatz ist. Wichtig ist, dass sich so ein Werkzeug in die vorhandene IT-Landschaft integrieren lässt. Das kann beispielsweise mit OSLC, einem von IBM geschaffenen Standard zur Kopplung von IT-Systemen, erfolgen.

Herr Dr. Otterbach, ich bedanke mich für das Gespräch.

INTERVIEW: Markus Schöttle FOTO: dSpace 